\title{
Time-Reversal Optical Focusing for Biophotonics Applications
}

\author{
Changhuei Yang \\ California Institute of Technology, 1200 E. California Blvd., MC 136-93, Pasadena, CA 91125
}

\begin{abstract}
I will discuss our recent work on the use of digital optical phase conjugation and ultrasound tagging to accomplish timereversal deep tissue optical focusing for fluorescence imaging and other applications.
\end{abstract}

\section{KEYWORD LIST}

phase-conjugation, optical time-reversal, deep-tissue imaging

One of the primary challenges in biophotonics is that it is not possible to focus light tightly in deep tissues using conventional optics. Much like the case of fog, tissue turbidity obscures the line of sight by diffusing light and preventing the formation of an optical focus. The extent of tissue scattering is remarkably high - as a point of reference, the mean scattering length of $633 \mathrm{~nm}$ light in dermis is $\sim 50$ microns ${ }^{[1]}$. Although advances such as multiphoton excitation have enabled greater optical penetration, these strategies are still fundamentally limited to superficial depths. The most mature of these strategies - two photon excitation, can hardly access depths beyond $1 \mathrm{~mm}{ }^{[2,3]}$. This is because these methods disregard scattered light as noise, depending only on the ballistic (non-scattered) photons whose population depletes exponentially with depth. Therefore, optical focusing, and techniques that rely on optical focusing, has long been considered impossible beyond a depth of $1 \mathrm{~mm}$.

Interestingly, optimally delivering light through a scattering medium is not an impossible proposition. Simplistically speaking, if we have full knowledge of the positions and scattering profile of the scatterers within the scattering medium, it would be possible to tailor an incident wavefront to optimally couple light to any specific point within the tissue. In other

(a)

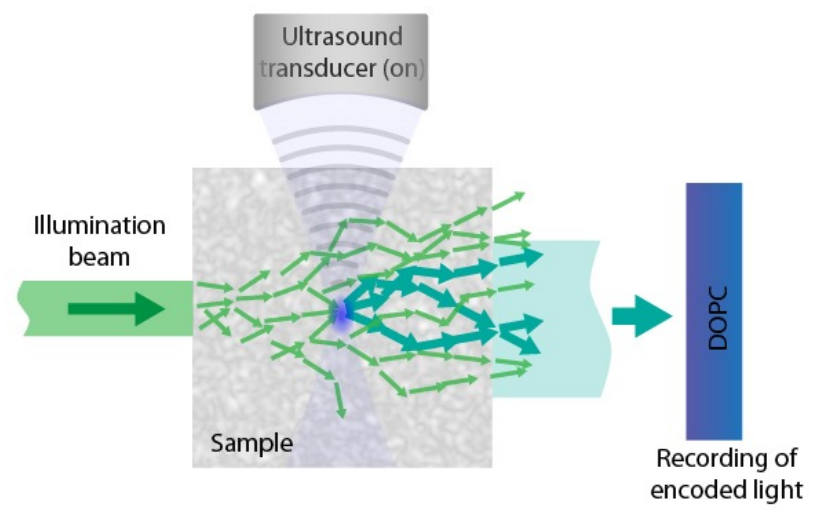

(b)

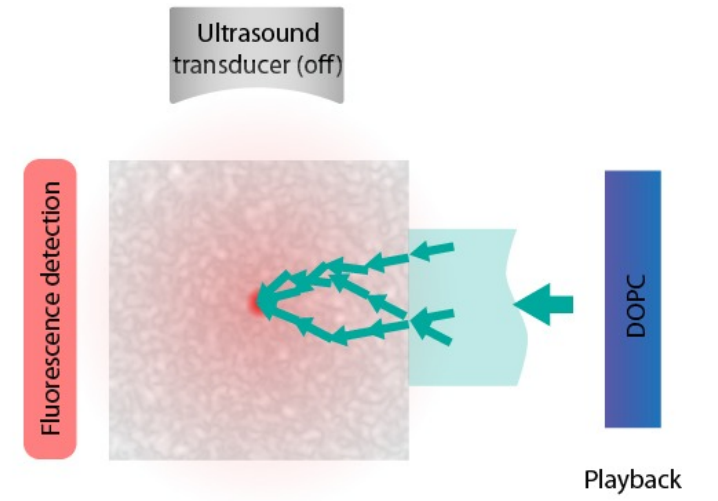

Fig. 1 | Principles of DOPC-TRUE. a, We use an ultrasound transducer (frequency $=f$ ) to focus acoustic power into a desired spatial point. The DOPC selectively records the light field components (upshifted by f) that have passed through that focal point. b, The DOPC then generates a light field that must pass through that focal point - effectively focusing light onto that point. This focal point can be used to excite fluorophores or other intrinsic biomedical optical signatures. 
words, we can capitalize on the fact that scattering is a deterministic process to precisely manipulate light within a scattering medium. One way to achieve this is by optical time reversal (also known as optical phase conjugation). The basic premise of time reversal is that the scattered wavefront contains information about the scattering medium, such that when it is time-reversed or phase conjugated through the same medium, it predictably undistorts to recover the original unscattered wavefront.

By exploiting the time-reversal symmetry of optical scattering, we can develop methods to make use of the

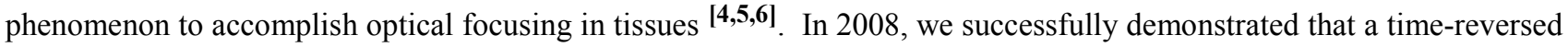
scattered field can force light to retrace its paths through a scattering ex vivo tissue and resulting in the unscrambling of the original light field pattern ${ }^{[5]}$. This opened up the interesting proposition of undoing tissue scattering to accomplish optical focusing through tissues. The use of time reversal for focusing and controlling light inside tissue was first reported by Lihong Wang's group in 2011. By combining the time-reversal property of light propagation with ultrasound-tagging, the group achieved a sub-millimeter scale optical focus within a tissue phantom; the technique was named time-reversal enabled ultrasound (TRUE) focusing ${ }^{[7]}$.

The working principle of TRUE is the following (Fig. 1): Unlike light, ultrasound focusing is possible in tissues because acoustic waves are not significantly scattered. Part of the scattered optical wavefront that passes through an ultrasound focus gets frequency-shifted through the acousto-optic effect. Thus, the ultrasound focus essentially becomes a virtual source of frequency-shifted (or, "ultrasound-tagged") light. The ultrasound-tagged light can easily be detected via interferometric methods and time-reversed by a time reversal mirror to form an optical focus at the location of the ultrasound focus. In its original implementation with the use of photorefractive crystal as the time reversal mirror, the generated TRUE focus is relatively low intensity and thus only allowed absorption characterization of the sub-millimeter scale focused spot ${ }^{[7]}$.

We recently showed that the use of an optoelectronic time reversal technique (digital optical phase conjugation; DOPC) with TRUE enabled unprecedented high resolution focusing and fluorescence imaging, $2.5 \mathrm{~mm}$ deep in ex vivo tissue ${ }^{[8]}$. The incorporation of the DOPC enable time reversal with high gain, which is challenging for photorefractive crystal based time reversal mirrors.

This is because the ultrasound-tagging efficiency in tissues is low; only $10^{-2}$ of light that passes through the ultrasound focus will be tagged. Since only a fraction of the diffused beam passes through the ultrasound focus, the total population of tagged versus untagged light exiting the tissue in our experiments was $10^{-4}[8]$, a fraction that would decrease with increasing depth. To form a time-reversed focus of sufficient intensity, a large phase conjugate optical gain would be required. This cannot be easily achieved using traditional phase conjugate mirrors, which have typical gains of less than unity ${ }^{[9]}$. However, this requirement can be easily met with DOPC. In our experiments, we selectively measured and phase conjugated ultrasound frequency-tagged light with $\sim 5 \times 10^{5}$ gain ${ }^{[8]}$. We note that we would be able to achieve even higher gain simply by increasing the playback laser power.

Because perfect time reversal requires control over phase, amplitude and polarization of the entire scattered field (propagating and evanescent), experimental time reversal is practically always incomplete and is accompanied by a diffuse background ${ }^{[10]}$. The focal peak to background ratio (PBR) is determined by the number of discrete spatial controls we have over the scattered wavefront $(\mathrm{N})$, which is equivalent to the maximum number of statistically independent optical speckles that are measured and played-back; and the number of optical modes modulated by the ultrasound focus (M), such that PBR $\sim \mathrm{N} / \mathrm{M}^{[10]}$. In our experiments, we achieved an PBR of $5.5^{[8]}$. 


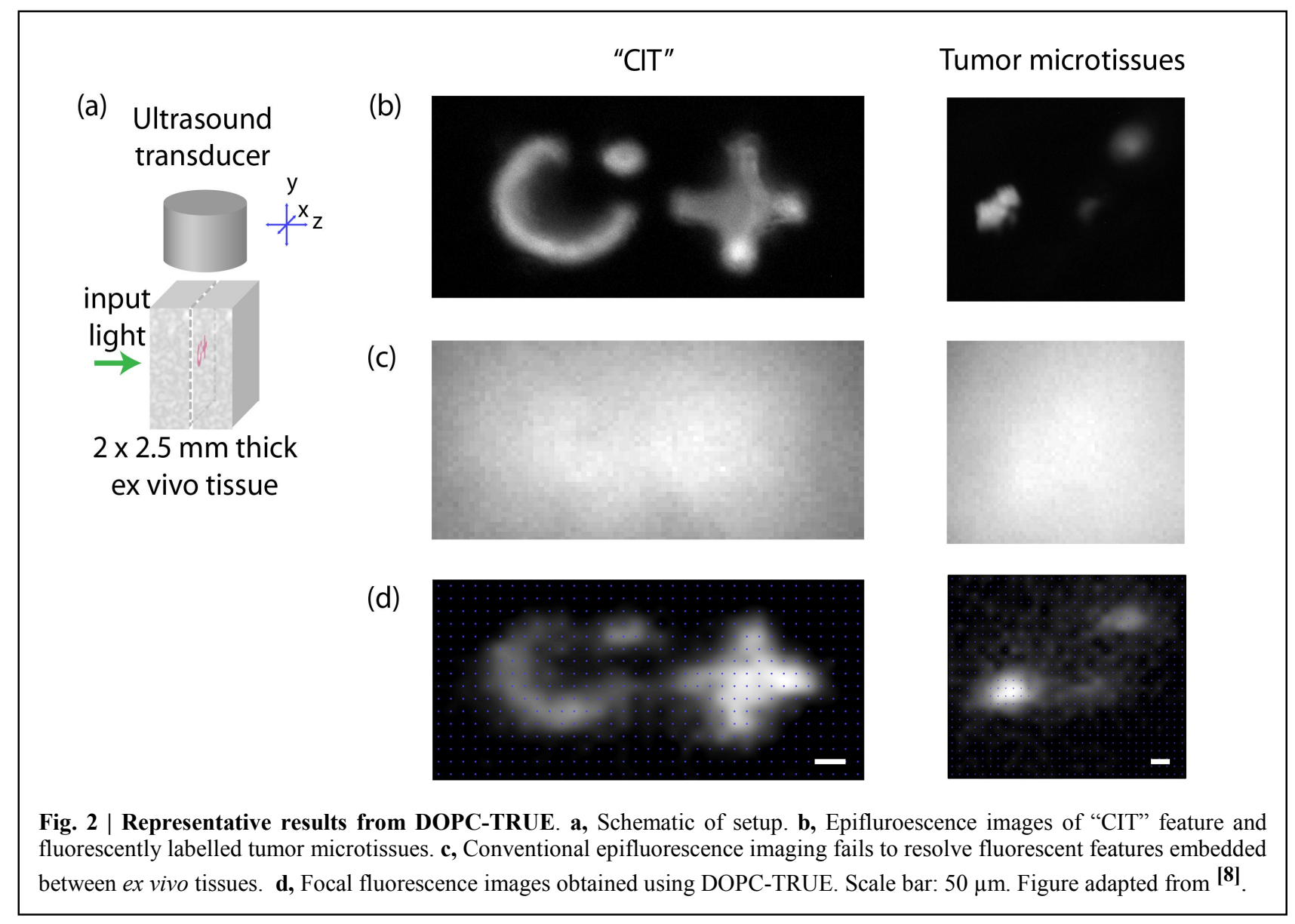

This advancement with digital TRUE allowed high intensity focusing and focal fluorescence imaging at a resolution of 36 by $52 \mu \mathrm{m}, 2.5 \mathrm{~mm}$ deep in ex vivo tissues (Fig. 2) ${ }^{[8]}$. Recently, using a modified method, we further showed that focusing to a spot size of $5 \mu \mathrm{m}(\sim 1 / 6$ of the ultrasound focal width $)$ is possible ${ }^{[11]}$. Beyond fluorescence imaging, our demonstration as described in the last reference [12] paves the way for many more biomedical applications in deep tissues.

\section{ACKNOWLEDGMENTS}

This work is supported by NIH 1DP2OD007307-01 and R21 EB012255 as well as DARPA W31P4Q-11-1-0008. Benjamin Judkewitz is a recipient of a Sir Henry Wellcome Postdoctoral Fellowship by the Wellcome Trust. Ying Min Wang acknowledges support from the National Science Scholarship, awarded by the Agency for Science, Technology and Research, Singapore. 


\section{REFERENCES}

[1] Cheong, W.F., S.A. Prahl, and A.J. Welch, "A review of the optical properties of biological tissues," IEEE Journal of Quantum Electronics 26(12), 2166-2185 (1990).

[2] Denk, W., J.H. Strickler, and W.W. Webb, “2-Photon Laser Scanning Fluorescence Microscopy," Science 248(4951), 73-76 (1990).

[3] Helmchen, F. and W. Denk, “Deep tissue two-photon microscopy,” Nature Methods 2(12), 932-940 (2005).

[4] McDowell, E.J., M. Cui, I.M. Vellekoop, V. Senekerimyan, Z. Yaqoob, C. Yang, "Turbidity suppression from the ballistic to the diffusive regime in biological tissues using optical phase conjugation," Journal of Biomedical Optics 15(2), 025004-025004 (2010).

[5] Yaqoob, Z., D. Psaltis, M. S. Feld, C. Yang, "Optical phase conjugation for turbidity suppression in biological samples," Nature Photonics 2(2), 110-115 (2008).

[6] Cui, M. and C. Yang, "Implementation of a digital optical phase conjugation system and its application to study the robustness of turbidity suppression by phase conjugation," Optics Express 18(4), 3444-3455 (2010).

[7] Xu, X., H. Liu, and L.V. Wang, “Time-reversed ultrasonically encoded optical focusing into scattering media," Nature Photonics 5(3), 154-157 (2011).

[8] Wang, Y.M., B. Judkewitz, C. A. DiMarzio, C. Yang, "Deep-tissue focal fluorescence imaging with digitally timereversed ultrasound-encoded light," Nature Communications 3, Article number 928 (2012).

[9] Gunter, P., J. P. Huignard, [Photorefractive Materials and their Applications 1: Basic Effects], Springer (2006).

[10] Vellekoop, I.M., "Controlling the propagationg of light in disordered scattering media," Thesis, University of Twente (2009).

[11] Judkewitz, B., Y. M. Wang, R. Horstmeyer, A. Mathy, C. Yang, "Speckle-scale focusing in the diffusive regime with time-reversal of variance-encoded light (TROVE)," Nature Photonics 7(4), 300-305 (2013).

[12] Tye, K.M., R. Prakash, S-Y. Kim, L.E. Fenno, L. Grosenick, H. Zarabi, K.R. Thompson, V. Gradinaru, C. Ramakrishnan, K. Deisseroth, "Amygdala circuitry mediating reversible and bidirectional control of anxiety," Nature 471(7338), 358-62 (2011). 